\title{
Simulation of Neurotransmitter Transport in a Presynaptic Bouton of a Neuron
}

\author{
BARTŁOMieJ SiweK ${ }^{1}$ \\ ${ }^{1}$ Institute of Computer Science, Jagiellonian University, \\ Prof. Stanisława Łojasiewicza 6, 30-348 Cracow, Poland \\ e-mail: siwek@ii.uj.edu.pl
}

\begin{abstract}
In this paper the results of numerical simulation of neurotransmitter fast transport in a presynaptic bouton of a biological neuron are presented. A mathematical model governing the transport that is fully described in [2] is recalled. A numerical simulation scheme, used parameters and their origins are described. Finally, the results of the simulation are presented.
\end{abstract}

Keywords: numeric simulation, Galerkin method, fast transport, neurotransmitter, presynaptic bouton.

\section{Introduction}

Neurotransmitter fast transport in the axon terminal of a presynaptic bouton is one of the primary mechanisms underlying the nerve system operation. This process is usually modeled using various methods - probabilistic methods, ordinary differential equations or partial differential equations. A synthesis, transport and release of a neurotransmitter model based on the partial differential equations was shown in [2]. This paper describes the numerical approach used to simulate the aforementioned model and presents the preliminary results of this simulations. 


\section{Mathematical model of fast transmission}

Biological foundations. A fast component of synaptic transmission is a process of small molecules (glutamate, acetylcholine, GABA) release from the docked synaptic vesicles into the synaptic cleft. The molecules are synthesized in the axon terminal of the presynaptic neuron. The release takes place after the arrival of the action potential to the terminal button. The presented model describes the refill of the vesicle pool in the docking site. It is assumed that this process is a simple diffusion - vesicles diffuse in the cytoplasm and dock in the specific regions of the cell membrane. The process takes place in the time scale of the range $1 \mathrm{~ms}$. The biological assumptions both mentioned above and specified in the sequel are based on $[1,5,6,8,9,10]$.

\section{Model assumptions.}

1. The terminal button occupies a fixed domain, a fixed part of the domain boundary are the vesicle release sites.

2. The unknown of the model is the concentration of vesicles in the cytoplasm. The unit in which this value is expressed can either be the mass or the quantity of the vesicles or the fraction of the cytoplasm volume they occupy.

3. Vesicles diffuse inside the terminal button and they are synthesized in some subdomain of the button.

4. The efficiency of the vesicle synthesis may either be assumed to be constant or proportional to the difference between the equilibrium concentration (above which the synthesis does not take place) and current concentration.

5. Vesicles do not leave the domain unless the action potential arrives. The arrival of the action potential triggers the possibility of the vesicles release through some fixed period of time. The number of vesicles that can be released in a time unit through the unit area is proportional to the vesicle concentration in the vicinity of the release site.

\section{Model parameters.}

(i) $\Omega \subset \mathbb{R}^{3}$ - the domain of the terminal button,

(ii) $\Omega_{1} \subset \Omega$ - the domain of the neurotransmitter production,

(iii) $\partial \Omega_{d} \subset \partial \Omega$ - neurotransmitter release sites on the cell membrane, 
(iv) $f: \Omega \rightarrow \mathbb{R}$ - the neurotransmitter source density defined, for example, by $f(x)=0$ outside $\Omega_{1}$ and $f(x)=f_{z}$ on $\Omega_{1}$,

(v) $\bar{\rho}$ - the balance concentration of the neurotransmitter in the button,

(vi) $\alpha$ - the coefficient denoting the rate of neurotransmitter exocytosis, $\alpha$ is the number of vesicles (or molecules) which are released through the unit area of the membrane in a time unit by the unit difference of the concentration in the cell and outside the cell (1 action potential activates 300 vesicles and 1 vesicle contains 1 000-10 000 molecules of the neurotransmitter),

(vii) $a_{i j}: \Omega \rightarrow \mathbb{R}$ - the diffusion tensor for the neurotransmitter (assuming that the cytoplasm is an isotropic and homogeneous medium the tensor is diagonal and constant in time; the value of all three entries on the diagonal is constant and equal to the diffusion coefficient which, for the acetylcholine, is equal to $300 \mu \mathrm{m}^{2} / \mathrm{s}$ - see [3]),

(viii) $\tau$ - the time period through which the neurotransmitter is released from the docked vesicles to the cleft $(2-5 \mu s)$,

(ix) $t_{0}$ - the arrival moment of the potential.

Governing equations. The unknown in the model is the function $\rho: \Omega \times[0, T] \rightarrow \mathbb{R}$ denoting the concentration of the vesicles with the neurotransmitter.

The function is the solution to the equation

$$
\frac{\partial \rho(x, t)}{\partial t}=\sum_{i, j=1}^{3} \frac{\partial}{\partial x_{i}}\left(a_{i j}(x) \frac{\partial \rho(x, t)}{\partial x_{j}}\right)+f(x)(\bar{\rho}-\rho(x, t))^{+} .
$$

Remark: The first term on the right hand side of the equation is the classical diffusive term. The second one denotes the neurotransmitter production modelled by the function $f$. The production is weighed by the term $(\bar{\rho}-\rho(x, t))^{+}$and in consequence the neurotransmitter is not produced if its concentration is greater than $\bar{\rho}$. This term yields the equation nonlinear. To cope with the nonlinearity it is possible either to remove the weighting term completely or to test its value in some time points and assume that it is constant on the intervals between these points. This approximation will give the equation which is piecewise linear and for small interval lengths it will be good approximation of the nonlinear problem if the concentration does not change fast.

The boundary conditions are defined in the following way: 
- On the whole boundary of $\Omega$ apart from the docking sites we assume that no vesicles leave the cytoplasm, which leads to the homogeneous Neumann boundary condition

$$
\sum_{i, j=1}^{3} a_{i j} \frac{\partial \rho(x, t)}{\partial x_{j}} n_{i}=0 \quad \text { for } \quad(x, t) \in\left(\partial \Omega-\partial \Omega_{d}\right) \times[0, T] .
$$

- Similarly at the docking sites there is no flow of vesicles apart from the time interval on which the voltage dependent calcium channels are open after the arrival of the action potential

$$
\sum_{i, j=1}^{3} a_{i j} \frac{\partial \rho(x, t)}{\partial x_{j}} n_{i}=0 \quad \text { for } \quad(x, t) \in \partial \Omega_{d} \times\left(\left[0, t_{0}\right) \cup\left(t_{0}+\tau, T\right]\right) .
$$

- At the docking sites within the time interval on which ion channels are open (which is triggered by the action potential) the flow of the vesicles outside the button is proportional to their concentration inside the domain. We assume that the vesicle concentration outside $\Omega$ is equal to 0 .

$$
\sum_{i, j=1}^{3} a_{i j} \frac{\partial \rho(x, t)}{\partial x_{j}} n_{i}=\alpha \rho(x, t) \quad \text { for } \quad(x, t) \in \partial \Omega_{d} \times\left[t_{0}, t_{0}+\tau\right] .
$$

We also assume the initial condition

$$
\rho(x, 0)=\rho_{0}(x) \quad \text { on } \quad \Omega .
$$

Weak formulation. Problem well posedness. The unknown concentration is sought in the space $\mathcal{V}=\left\{v \in L^{2}(0, T, V), v^{\prime} \in L^{2}\left(0, T ; V^{*}\right)\right\}$, where $V=H^{1}(\Omega)$, and $V^{*}$ is its dual space (with the duality pairing denoted by $\langle\cdot, \cdot\rangle)$. A scalar product in $L^{2}(\Omega)$ is denoted by $(\cdot, \cdot)$.

The weak formulation of the problem that governs the phenomenon is given as follows (see [2], Eq. (10)):

Find $\rho \in \mathcal{V}$ such that

$$
\begin{aligned}
& \left\langle\frac{d \rho(t)}{d t}, v\right\rangle+\sum_{i, j=1}^{3}\left(a_{i j} \frac{\partial \rho(t)}{\partial x_{j}}, \frac{\partial v}{\partial x_{i}}\right)+\int_{\Omega_{d}} \alpha(t) \rho(t) v d \sigma= \\
& =\left(f \cdot\left(\bar{\rho}-\rho_{n}(t)\right)^{+}, v\right) \text { for } v \in V \text { a.e. } t \in(0, T), \\
& \rho(0)=\rho_{0} \in L^{2}(\Omega),
\end{aligned}
$$


where $\alpha(t)=\alpha$ for $t \in\left[t_{0}, t_{0}+\tau\right]$ (there are possibly more subintervals during which the terminal excitation takes place) and $\alpha(t)=0$ otherwise. Diffusion tensor $a_{i j}$ and excitation rate $f$ are possibly dependent on $x$ (that represents heterogeneity and anisotropy of the bouton domain). If we introduce the linear operator $A(t): V \rightarrow V^{*}$ given by $\langle A(t) u, v\rangle=\sum_{i, j=1}^{3}\left(a_{i j} \frac{\partial u}{\partial x_{j}}, \frac{\partial v}{\partial x_{i}}\right)+$ $\int_{\Omega_{d}} \alpha(t) u v d \sigma$ and the nonlinear operator $B: V \rightarrow V^{*}$ given by $\langle B u, v\rangle=$ $\left(f \cdot(\bar{\rho}-u)^{+}, v\right)$, then the equation (6) takes the simple form

$$
\frac{\partial \rho(t)}{\partial t}+A(t) \rho(t)=B \rho(t) \text { in } V^{*} \text { for a.e. } t \in(0, T) \text {. }
$$

In [2] (see Theorem 1) it is shown, that, under appropriate reasonable assumptions on the problem data, it has exactly one solution.

\section{Numerical simulations}

This section presents the tools and methods used for simulating neurotransmitter fast transport. We begin by presenting a mathematical derivation of the iterative method implemented in the simulation software. This software complemented by the mesh generator and results visualizer constitutes a full software package used. The obtained results are presented in the concluding part of this section.

\subsection{Finite element method}

Solving partial differential equations in the three dimensional space suffers from many problems - generation of a three dimensional mesh or spatial integration. Because of all these reasons the presented model was narrowed to two dimensions.

Let us assume that we have a mesh of Lagrange elements. Let $x_{i}$ be a mesh point for $i=1, \ldots, n$. By $\Delta_{i j k}$ we will denote a Lagrange element with vertices in $x_{i}, x_{j}$ and $x_{k}$ points. Let $v_{i}$ be linear test functions for $i=1, \ldots, n$. In order to define the functions formally we will choose $i=1, \ldots, n$ and $j, k \in\{1, \ldots, n\}$, such that $x_{i}, x_{j}, x_{k}$ points define a Lagrange element. Then the $v_{i}$ function is defined as follows:

$$
v_{i}: \mathbb{R}^{2} \ni(x, y) \rightarrow\left\{\begin{array}{ll}
\frac{\left(x_{j}^{1}-x\right)\left(x_{k}^{2}-y\right)-\left(x_{j}^{2}-y\right)\left(x_{k}^{1}-x\right)}{\left(x_{j}^{1}-x_{i}^{1}\right)\left(x_{k}^{2}-x_{i}^{2}\right)-\left(x_{j}^{2}-x_{i}^{2}\right)\left(x_{k}^{1}-x_{i}^{1}\right)} & :(x, y) \in \Delta_{i j k} \\
0 & : \text { otherwise }
\end{array} \in \mathbb{R} .\right.
$$


The geometrical interpretation of this function is very simple - it is an area of the triangle with vertices in $(x, y), x_{j}, x_{k}$ divided by the area of the $x_{i}, x_{j}, x_{k}$ triangle. Using the triangle area formula based on the vector product in $\mathbb{R}^{2}$ and simplifying it we obtain the above result. The important property of this definition is

$$
v_{i}\left(x_{j}\right)=\left\{\begin{array}{ll}
1 & : i=j \\
0 & : i \neq j
\end{array} .\right.
$$

Having defined the test functions we can now present the Finite Element Method used to solve the equation (6). We approximate the $\rho$ function using linear combinations of test functions with time dependent coefficients. We assume

$$
\rho(x, t)=\sum_{l=1}^{n} z_{l}(t) v_{l}(t), \quad \text { where } \quad z_{l}:[0, T] \ni t \rightarrow \mathbb{R} .
$$

Using the above equation, we substitute $\rho$ in (6) obtaining

$$
\begin{gathered}
\left(\frac{\partial}{\partial t}\left(\sum_{l=1}^{n} z_{l}(t) v_{l}(x)\right), v(x)\right)=-\sum_{i, j=1}^{2}\left(a_{i j}(x) \frac{\partial}{\partial x_{j}}\left(\sum_{l=1}^{n} z_{l}(t) v_{l}(x)\right), \frac{\partial v}{\partial x_{i}}(x)\right)- \\
\cdots-\int_{\partial \Omega} \alpha(t) \sum_{l=1}^{n} z_{l}(t) v_{l}(x) v(x) d \sigma+\left(\beta(x)\left(\bar{\rho}-\sum_{l=1}^{n} z_{l}(t) v_{l}(x)\right)^{+}, v(x)\right) .
\end{gathered}
$$

Simplifying the equation, we get

$$
\begin{aligned}
& \sum_{l=1}^{n} \frac{\partial z_{l}}{\partial t}(t)\left(v_{l}(x), v(x)\right)=-\sum_{i, j=1}^{2} \sum_{l=1}^{n} z_{l}(t)\left(a_{i j}(x) \frac{\partial v_{l}}{\partial x_{j}}(x), \frac{\partial v}{\partial x_{i}}(x)\right)-\ldots \\
& \cdots-\alpha(t) \sum_{l=1}^{n} z_{l}(t) \int_{\partial \Omega} v_{l}(x) v(x) d \sigma+\left(\beta(x)\left(\bar{\rho}-\sum_{l=1}^{n} z_{l}(t) v_{l}(x)\right)^{+}, v(x)\right) .
\end{aligned}
$$

Since all the above transformations hold for all $v \in C^{\infty}(\Omega)$ we assume $v$ to be $v_{1}, v_{2}, \ldots, v_{n}$, thus obtaining a following equation system

$$
\begin{gathered}
\sum_{l=1}^{n} \frac{\partial z_{l}}{\partial t}(t)\left(v_{l}(x), v_{k}(x)\right)=-\sum_{i, j=1}^{2} \sum_{l=1}^{n} z_{l}(t)\left(a_{i j}(x) \frac{\partial v_{l}}{\partial x_{j}}(x), \frac{\partial v_{k}}{\partial x_{i}}(x)\right)-\ldots \\
\cdots-\alpha(t) \sum_{l=1}^{n} z_{l}(t) \int_{\partial \Omega} v_{l}(x) v_{k}(x) d \sigma+\left(\beta(x)\left(\bar{\rho}-\sum_{l=1}^{n} z_{l}(t) v_{l}(x)\right)^{+}, v_{l}(x)\right),
\end{gathered}
$$


for all $k=1, \ldots, n$. The matrix representation of the above system is

$$
\begin{aligned}
& \underbrace{\left[\begin{array}{cccc}
\left(v_{1}, v_{1}\right) & \left(v_{2}, v_{1}\right) & \ldots & \left(v_{n}, v_{1}\right) \\
\left(v_{1}, v_{2}\right) & \left(v_{2}, v_{2}\right) & \ldots & \left(v_{n}, v_{2}\right) \\
\vdots & \vdots & \ddots & \vdots \\
\left(v_{1}, v_{n}\right) & \left(v_{2}, v_{n}\right) & \ldots & \left(v_{n}, v_{n}\right)
\end{array}\right]}_{G} \underbrace{\left[\begin{array}{c}
\frac{\partial z_{1}}{\partial t} \\
\frac{\partial z_{2}}{\partial t} \\
\vdots \\
\frac{\partial z_{l}}{\partial t}
\end{array}\right]}_{\frac{d z}{d t}(t)}= \\
& =\underbrace{\left[\begin{array}{ccc}
-\sum_{i, j=1}^{2}\left(a_{i j} \frac{\partial v_{1}}{\partial x_{j}}, \frac{\partial v_{1}}{\partial x_{i}}\right) & \ldots & -\sum_{i, j=1}^{2}\left(a_{i j} \frac{\partial v_{n}}{\partial x_{j}}, \frac{\partial v_{1}}{\partial x_{i}}\right) \\
\vdots & \ddots & \vdots \\
-\sum_{i, j=1}^{2}\left(a_{i j} \frac{\partial v_{1}}{\partial x_{j}}, \frac{\partial v_{n}}{\partial x_{i}}\right) & \ldots & -\sum_{i, j=1}^{2}\left(a_{i j} \frac{\partial v_{n}}{\partial x_{j}}, \frac{\partial v_{n}}{\partial x_{i}}\right)
\end{array}\right]}_{A} \underbrace{\left[\begin{array}{c}
z_{1} \\
z_{2} \\
\vdots \\
z_{l}
\end{array}\right]}_{z(t)}+ \\
& +\alpha(t) \underbrace{\left[\begin{array}{ccc}
-\int_{\partial \Omega} v_{1} v_{1} d \sigma & \ldots & -\int_{\partial \Omega} v_{n} v_{1} d \sigma \\
\vdots & \ddots & \vdots \\
-\int_{\partial \Omega} v_{1} v_{n} d \sigma & \ldots & -\int_{\partial \Omega} v_{n} v_{n} d \sigma
\end{array}\right]}_{B^{\prime}} \underbrace{\left[\begin{array}{c}
z_{1} \\
z_{2} \\
\vdots \\
z_{l}
\end{array}\right]}_{z(t)}+ \\
& +\underbrace{\left[\begin{array}{c}
\left(\beta(x)\left(\bar{\rho}-\sum_{l=1}^{n} z_{l}(t) v_{l}(x)\right)^{+}, v_{1}\right) \\
\vdots \\
\left(\beta(x)\left(\bar{\rho}-\sum_{l=1}^{n} z_{l}(t) v_{l}(x)\right)^{+}, v_{n}\right)
\end{array}\right]}_{P(z(t))} .
\end{aligned}
$$

The short version of the above matrix equation is as follows:

$$
G \frac{d z}{d t}(t)=A z(t)+\alpha(t) B^{\prime} z(t)+P(z(t)) .
$$

Since we are using a fixed mesh and presented test functions the values of $G, B^{\prime}$ and $A$ matrices depend only on the spatial variable, thus it is possible to compute them at this point. In the implementation used, the Gauss points method is used for this task. Furthermore, from the above formula we can observe, that

$$
\left[\begin{array}{c}
\rho_{0}\left(x_{1}\right) \\
\rho_{0}\left(x_{2}\right) \\
\vdots \\
\rho_{0}\left(x_{n}\right)
\end{array}\right]=\left[\begin{array}{c}
\rho\left(0, x_{1}\right) \\
\rho\left(0, x_{2}\right) \\
\vdots \\
\rho\left(0, x_{n}\right)
\end{array}\right]=\left[\begin{array}{c}
\sum_{l=1}^{n} z_{l}(0) v_{l}\left(x_{1}\right) \\
\sum_{l=1}^{n} z_{l}(0) v_{l}\left(x_{2}\right) \\
\vdots \\
\sum_{l=1}^{n} z_{l}(0) v_{l}\left(x_{n}\right)
\end{array}\right]=\left[\begin{array}{c}
z_{1}(0) \\
z_{2}(0) \\
\vdots \\
z_{l}(0)
\end{array}\right]=z(0) .
$$


The last equality is obtained by the properties of the test functions $v_{i}$ for $i=1, \ldots, n$. Using a Crank-Nicholson method with $\Delta t$ time step, we obtain:

$$
\begin{gathered}
G \frac{z(t+\Delta t)-z(t)}{\Delta t}=\frac{1}{2}\left(A+\alpha(t+\Delta t) B^{\prime}\right) z(t+\Delta t)-\frac{1}{2} P(z(t)) \\
-\frac{1}{2}\left(A+\alpha(t) B^{\prime}\right) z(t)+\frac{1}{2} P(z(t+\Delta t)) .
\end{gathered}
$$

The result is an ordinary non-linear equation system. In order to solve it, we use a following iterative method - at the beginning we assume $z^{(0)}(t+\Delta t)=$ $z(t)$. We will solve the following equation system, at each step

$$
\begin{aligned}
G \frac{z^{(k+1)}(t+\Delta t)-z(t)}{\Delta t} & =\frac{1}{2}\left(A+\alpha(t+\Delta t) B^{\prime}\right) z^{(k+1)}(t+\Delta t)-\frac{1}{2} P(z(t)) \\
& -\frac{1}{2}\left(A+\alpha(t) B^{\prime}\right) z(t)+\frac{1}{2} P\left(z^{(k+1)}(t+\Delta t)\right) .
\end{aligned}
$$

However, the above equation system is still non-linear. In order to solve it, we substitute the $z^{(k+1)}(t+\Delta t)$ in the $P\left(z^{(k+1)}(t+\Delta t)\right)$ term with $z^{(k)}(t+\Delta t)$. As the result we obtain the following iterative method (for a fixed time point)

$$
\begin{aligned}
z^{(0)}(t+\Delta t) & =z(t) \\
G \frac{z^{(k+1)}(t+\Delta t)-z(t)}{\Delta t} & =\frac{\left(A+\alpha(t+\Delta t) B^{\prime}\right) z^{(k+1)}(t+\Delta t)}{2}-\frac{P(z(t))}{2} \\
& +\frac{P\left(z^{(k)}(t+\Delta t)\right)}{2}-\frac{\left(A+\alpha(t) B^{\prime}\right) z(t)}{2} \quad \text { for } k>1 .
\end{aligned}
$$

As a result in each step of this method a linear equation system is solved. In the simulation software implementation a bcgstab method, provided by the SciPy library for Python, was used.

\subsection{Mesh generation}

In order to facilitate the mesh generation, a separate program was created. Together with the Triangle library it constitutes a full solution for generation of meshes with the required parameters. The constructed software allows for defining the basic geometry that is further used for the mesh generation. This is made possible by allowing the user to define points, connect them into segments and place markers on the points, segments and areas that are used by the Finite Method Implementation for its purposes. These attributes allow us to define the neurotransmitter production domain and release sites. The defined geometry is then passed into the Triangle library, which processes it using an improved version of the Delaunay algorithm - the Ruppert 
algorithm. This allows us to generate a required triangular mesh without the losses in assigned parameters - they are transfered or interpolated onto newly created points. Additional control over the mesh parameters is obtained using the Triangle library option allowing to specify a lower bound on the triangle inner angles. This guarantees the stability of the numerical method used. The mesh used for simulations is presented below.

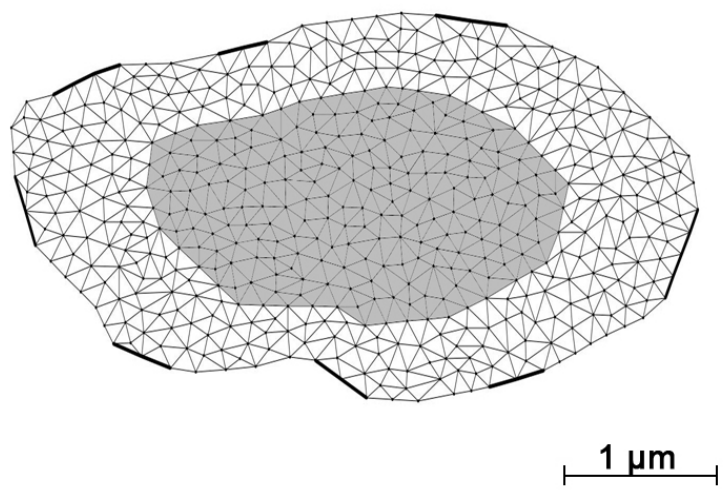

Fig. 1. A mesh

\subsection{Simulation parameters}

Below we present a table containing the values of parameters used for the simulation. The values were chosen to reassemble the conditions found in the nerve cells of Drosophila Melanogaster. The stimulation frequency was chosen as in the experiment described in [1]. Spatial parameters were developed using the photograph of the Drosophila melanogaster presynaptic bouton cross-section presented in [7].

The release sites were also marked in the mentioned figure, which allows them to be precisely marked. The neurotransmitter production domain, due to the lack of information other than its central location, was chosen to be of the same shape as the bouton scaled down and placed in the center.

Since the free acetylocholine has a diffusion coefficient equal to $300 \frac{\mu m}{s}$, for simulation this value was assumed to be 3 orders of magnitude smaller. The reason for such a modification is to compensate for the difference in the diffusion between free acetylocholine and that packed into vesicles. 
Tab. 1. Values of parameters used for the simulation

\begin{tabular}{|c|l|}
\hline Parameter & Value \\
\hline$\Omega$ & The mesh presented in Figure 1 \\
\hline$m(\Omega)$ & $\approx 8.06 \mu m^{2}$ \\
\hline$\Omega_{d}$ & The bold segments in Figure 1 \\
\hline$m\left(\Omega_{d}\right)$ & $\approx 3.46 \mu m$ \\
\hline$\Omega_{3}$ & The gray area in Figure 1 \\
\hline$m\left(\Omega_{3}\right)$ & $\approx 3.02 \mu m^{2}$ \\
\hline$a_{11}, a_{22}$ & $10^{-3} \cdot 300 \frac{\mu m}{s}$ \\
\hline$a_{12}, a_{21}$ & 0 \\
\hline$\tau$ & $4 \cdot 10^{-4} s$ \\
\hline$t_{0}$ & $\bigcup_{j=1}^{5}\{j+0.025 s \cdot i+0.0125 s\}_{i=1}^{19} \cup\{j+0.05 s \cdot i+5.025 s\}_{i=1}^{9}$ \\
\hline$\rho_{0}$ & $\frac{84000}{m(\Omega)} \approx \frac{84000}{8.06} \approx 10423 \frac{1}{\mu m^{2}}$ \\
\hline $\bar{\rho}$ & $\frac{70000}{m\left(\Omega_{3}\right)} \approx \frac{70000}{3.02} \approx 23198 \frac{1}{\mu m^{2}}$ \\
\hline$\alpha$ & $\frac{300}{84000 \tau}=\frac{300}{0.0004 \cdot 84000} \approx 8.93 \frac{1}{\mu m \cdot s}$ \\
\hline$\beta$ & $10^{-3} \frac{\alpha m\left(\Omega_{d}\right)}{m\left(\Omega_{3}\right)} \approx 0.01024 \frac{1}{\mu m^{2} \cdot s}$ \\
\hline$T$ & $5 s$ \\
\hline$\Delta t$ & $10^{-4} s$ \\
\hline
\end{tabular}

The initial density $\rho_{0}$ was calculated assuming the uniform placement of the 84000 vesicles (according to [7]) over the whole bouton domain. The production threshold was calculated assuming the uniform placement of the 70000 vesicles, constituting the reserve pool, over the production domain $\Omega_{3}$.

According to [2] one action potential activates about 300 vesicles, which corresponds to change of the pool by $\frac{300}{84000}$ in time $\tau$. From this we obtain the value of $\alpha$. The production efficiency was chosen to be 3 orders of magnitude smaller than the value that would counterbalance the release of the neurotransmitter. The reason for such an assumption is to approximate the behavior of the biological neurons, which under the intense stimulation exhibit the depletion of the available vesicles and regenerate slowly.

The release time $\tau$ of the vesicles was chosen to be equal to $0.4 m s$, which corresponds to the time of the action potential in creatures of the Drosophila melanogaster size. The time moments when the neurotransmitter release occurs were chosen to be evenly placed for the first as well as the second part 
of each second of the simulation. This corresponds to a $40 \mathrm{~Hz}$ stimulation in the first part and $20 \mathrm{~Hz}$ stimulation in the second part of each time unit. The whole simulation time $T$ was set to $5 s$ which is the same value as used in the experiment described in [1]. Finally, the time step was chosen to be dense enough to embrace the whole phenomenon - the release of the neurotransmitter in particular.

\subsection{Simulation results}

Below we present the result of the simulation. The dark areas represent zero density, while the white areas represent the maximal possible density.

\section{Concluding remarks}

In this paper we mentioned a mathematical model of neurotransmitter fast transport, described the methods used for its simulation and presented the obtained preliminary results. The model was set in a biological context through the appropriate choice of parameters resembling the conditions found in the nerval cells of Drosophila melanogaster.

An important conclusion arising from the performed simulation is correctness of the method used - experimental as well as theoretical. The observed neurotransmitter vesicle dynamics is compliant with intuition and expectations set by the studies of biological cells. Furthermore, the simulations exhibit a pattern ressembling the neurotransmitter vesicle depletion observed in nature, thus further proving correctness of the methods used.

Finally, we would like to point out the problem of choosing the appropriate shape of the production domain as well as production efficiency. During the preparation of this paper no publication regarding the mentioned parameters was found. The software used for simulations allows for exploring the wide range of possibilities for choosing just the two mentioned parameters. Along with the possibility to modify other aspects of the neurotransmitter vesicle dynamics this allows us to study of a broad range of neuron cells of many species, thus further allowing the verification of the model. 


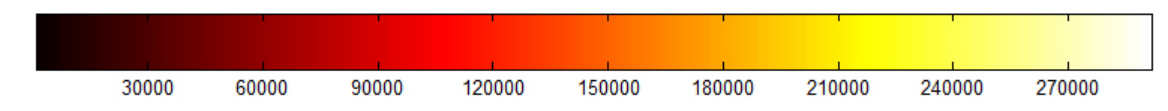

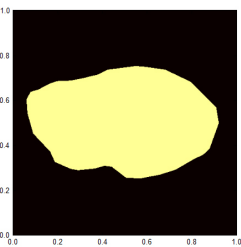

(a) $\mathrm{t}=0.0 \mathrm{~s}$

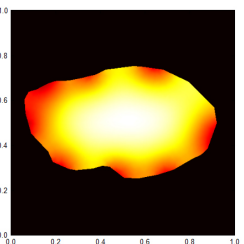

(a) $\mathrm{t}=0.625 \mathrm{~s}$

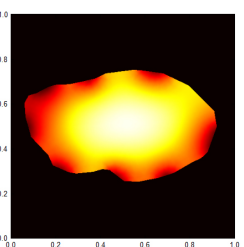

(a) $\mathrm{t}=1.25 \mathrm{~s}$

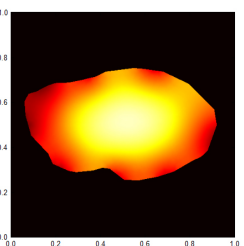

(a) $\mathrm{t}=1.875 \mathrm{~s}$

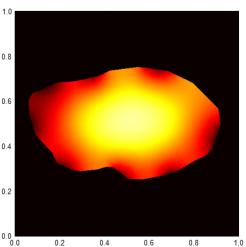

(a) $\mathrm{t}=2.5 \mathrm{~s}$

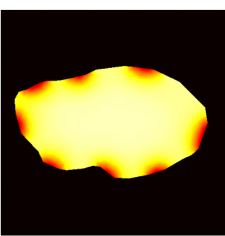

(b) $\mathrm{t}=0.125 \mathrm{~s}$

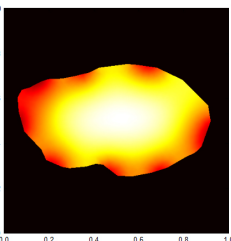

(b) $\mathrm{t}=0.75 \mathrm{~s}$

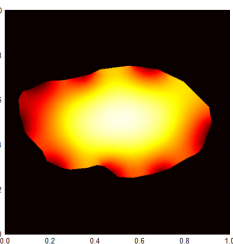

(b) $\mathrm{t}=1.375 \mathrm{~s}$

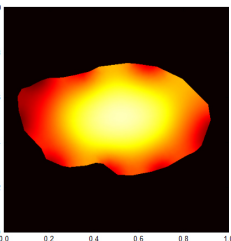

(b) $\mathrm{t}=2.0 \mathrm{~s}$

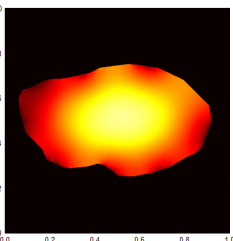

(b) $\mathrm{t}=2.625 \mathrm{~s}$

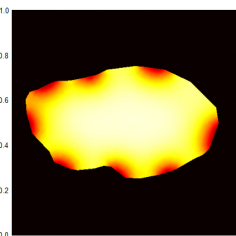

(c) $\mathrm{t}=0.25 \mathrm{~s}$

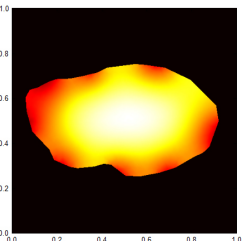

(c) $\mathrm{t}=0.875 \mathrm{~s}$

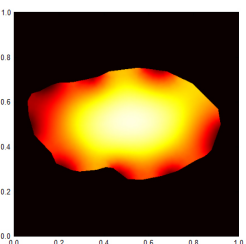

(c) $\mathrm{t}=1.5 \mathrm{~s}$

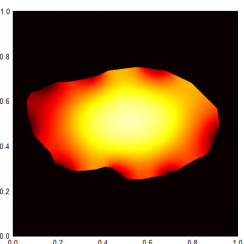

(c) $\mathrm{t}=2.125 \mathrm{~s}$

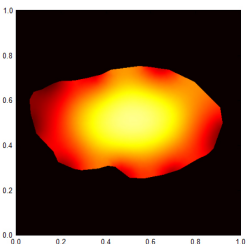

(c) $\mathrm{t}=2.75 \mathrm{~s}$

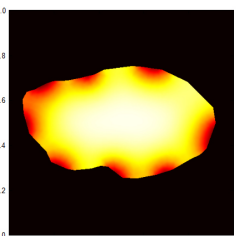

(d) $\mathrm{t}=0.375 \mathrm{~s}$

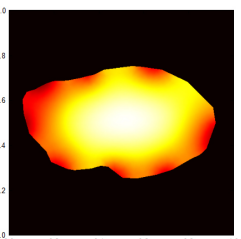

(d) $\mathrm{t}=1.0 \mathrm{~s}$

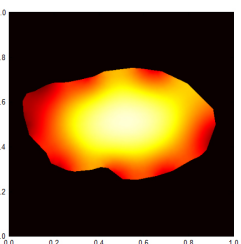

(d) $\mathrm{t}=1.625 \mathrm{~s}$

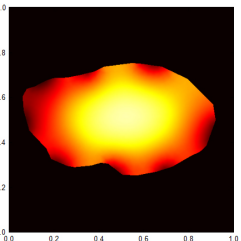

(d) $\mathrm{t}=2.25 \mathrm{~s}$

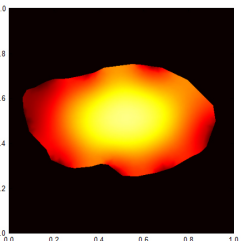

(d) $\mathrm{t}=2.875 \mathrm{~s}$

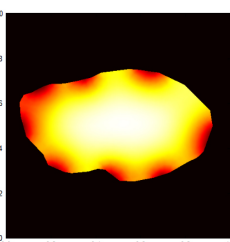

(e) $\mathrm{t}=0.5 \mathrm{~s}$

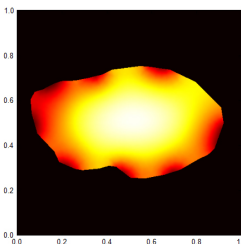

(e) $\mathrm{t}=1.125 \mathrm{~s}$

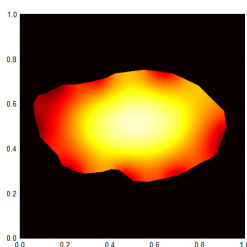

(e) $\mathrm{t}=1.75 \mathrm{~s}$

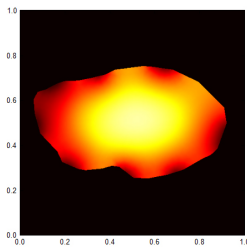

(e) $\mathrm{t}=2.375 \mathrm{~s}$

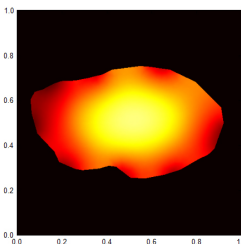

(e) $\mathrm{t}=3.0 \mathrm{~s}$ 


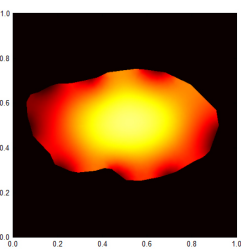

(a) $\mathrm{t}=3.0125 \mathrm{~s}$

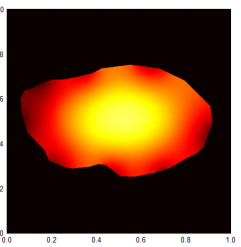

(a) $\mathrm{t}=3.75 \mathrm{~s}$

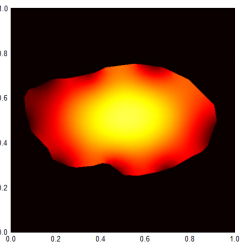

(a) $\mathrm{t}=4.375 \mathrm{~s}$

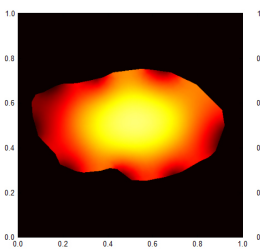

(b) $\mathrm{t}=3.25 \mathrm{~s}$

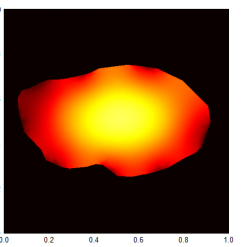

(b) $\mathrm{t}=3.875 \mathrm{~s}$

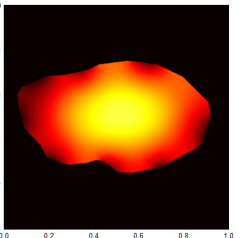

(b) $\mathrm{t}=4.5 \mathrm{~s}$

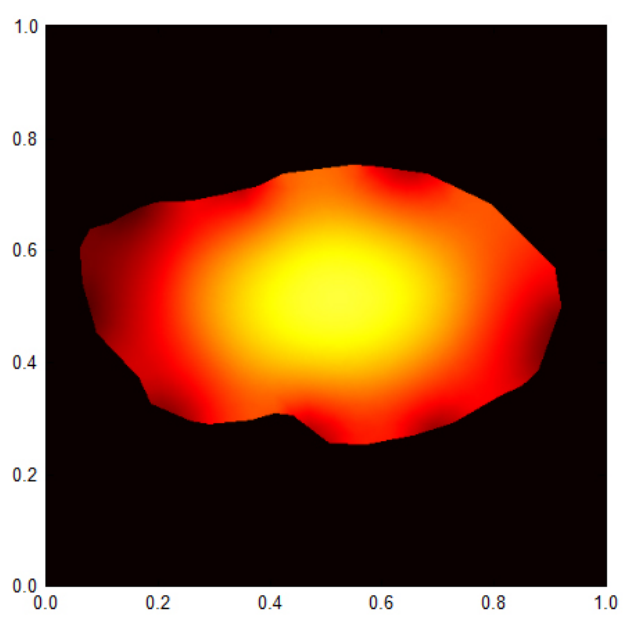

(f) $\mathrm{t}=5.0 \mathrm{~s}$

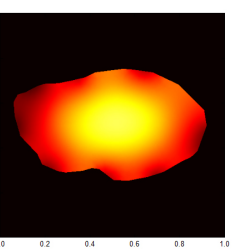

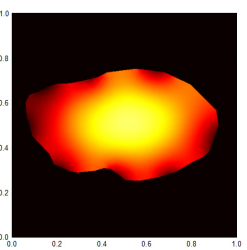

(d) $\mathrm{t}=3.5 \mathrm{~s}$

(c) $\mathrm{t}=4.0 \mathrm{~s}$

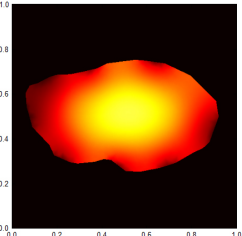

(c) $\mathrm{t}=4.625 \mathrm{~s}$

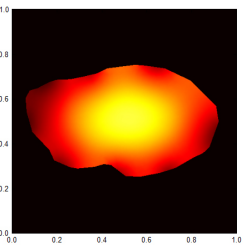

(d) $\mathrm{t}=4.75 \mathrm{~s}$

(d) $\mathrm{t}=4.125 \mathrm{~s}$ (e) $\mathrm{t}=3.625 \mathrm{~s}$

(e) $\mathrm{t}=4.25 \mathrm{~s}$

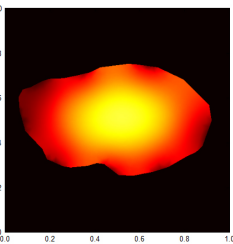

(e) $\mathrm{t}=4.875 \mathrm{~s}$
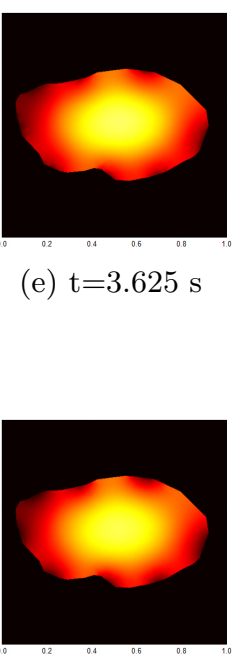


\section{References}

[1] Aristizabal F., Glavinovic M.I.; Simulation and parameter estimation of $d y$ namics of synaptic depression, Biological Cybernetics 90, 2004, pp. 3-18.

[2] Bielecki A., Kalita P.; Model of neurotransmitter fast transport in axon terminal of presynaptic neuron, Journal of Mathematical Biology 56, 2008, pp. 559-576.

[3] Chang S., Popov S.V.; Long-range signaling within growing neurites mediated by neurotrophin-3, Neurobiology 96, 1999, pp. 4095-4100.

[4] Magleby K.L., Stevens C.F.; A quantitative description of end-plate currents, Journal of Physiology 223, 1972, pp. 173-197.

[5] Oheim M., Loerke D., Stuhmer W., Chow R.H.; Multiple stimulation-dependent processes regulate the size of the relesable pool of vesicles, European Biophysics Journal 28, 1999, pp. 91-101.

[6] Parsons T.D., Coorsen J.R., Horstmann H., Almers W.; Docked granules, the exocytic burst, and the need for ATP hydrolysis in endoctrine cells, Neuron 15, 1995, pp. 1085-1096.

[7] Rizzoli S.O., Betz W.J.; Synaptic vesicle pools, Nature Reviews Neuroscience 6, 2005, pp. 57-69.

[8] Squire L.R. et al.; Fundamental Neuroscience, Academic Press, New York 2003.

[9] Steyer J.A., Horstmann H., Almers W.; Transport, docking and exocytosis of single granules in live chromaffin cells, Nature 388, 1997, pp. 474-478.

[10] Vitale M.L., Seward E.P., Trifaró J.M.; Chromaffin cell cortical actine network dynamics control the size of the release-ready vesicle pool and the initial rate of exocytosis, Neuron 14, 1995, pp. 353-363.

Received November 1, 2009 